\title{
Deposition and Transfer of Axonally Transported Phospholipids in Rat Sciatic Nerve
}

\author{
Arrel D. Toews, ${ }^{1,2}$ Regina Armstrong, ${ }^{2}$ Rita Ray, ${ }^{2}$ Robert M. Gould, ${ }^{3}$ and Pierre Morell ${ }^{1,2}$ \\ 'Department of Biochemistry and Nutrition, and 'Biological Sciences Research Center, University of North Carolina, \\ Chapel Hill, North Carolina 27514, and ${ }^{3}$ New York State Institute for Basic Research in Mental Retardation, Staten Island, \\ New York 10314
}

Radioactive glycerol, ethanolamine, or choline injected into the vicinity of the cell bodies of rat sciatic nerve sensory fibers is incorporated into phospholipid. Some newly synthesized ethanolamine and choline phosphoglycerides are subsequently committed to transport down the sciatic nerve axons at a rate of several hundred millimeters per day. Most labeled choline phosphoglycerides move uniformly down the axons; in contrast, the crest of moving ethanolamine phosphoglycerides is continually attenuated. These data, as well as differences in the clearance of these phospholipids distal to a nerve ligature, suggest that various classes of labeled phospholipids are differentially unloaded from the transport vector (possibly by exchange with unlabeled lipid in stationary axonal structures) during movement down the axons. The extent of unloading appears to be defined by the base moiety; both diacyl and plasmalogen species of ethanolamine phosphoglycerides exchange extensively with stationary axonal lipids, while most choline phosphoglycerides continue down the axons. Autoradiographic studies with ${ }^{3} \mathrm{H}$-choline and ${ }^{3} \mathrm{H}$-ethanolamine demonstrated that most unloaded phospholipid is initially deposited in axonal structures; some of this unloaded lipid is subsequently transferred to the axon/myelin interface (axolemma?) and then to myelin. Although transported ethanolamine phosphoglycerides exchange more extensively with lipids in stationary axonal structures than do choline phosphoglycerides, at early times more label from ${ }^{3} \mathrm{H}$-choline is found in myelin. A model to resolve this seeming discrepancy is proposed, wherein a differential topographic localization of phospholipid classes in the membrane of the transport vector allows for a preferential extensive exchange of transported ethanolamine phosphoglycerides with lipids in stationary axonal structures, while choline phosphoglycerides become available for rapid transfer to myelin by a process involving vesicle fusion with axolemma.

Axonal transport serves to maintain axons and nerve endings by providing them with a supply of necessary macromolecules synthesized in the neuronal perikarya (for reviews, see Grafstein

\footnotetext{
Received Mar. 20, 1987; revised July 16, 1987; accepted July 29, 1987.

This study was supported in part by USPHS Grants NS-1 1615, HD-03110, and NS-13980. R.A. is an NSF predoctoral fellow. We thank Julie Mason for final manuscript preparation, and Fred Connell and John Holshek for preparation of the photomicrographs.

Correspondence should bc addressed to Dr. Pierre Morell, 321 Biological Sciences Research Center $220 \mathrm{H}$, University of North Carolina, Chapel Hill, NC 27514.

Copyright (C) 1988 Society for Neuroscience $0270-6474 / 88 / 020593-09 \$ 02.00 / 0$
}

and Forman, 1980; Ochs, 1982; Weiss, 1982; Ledeen, 1985). Axonally transported macromolecules must be "unloaded" from the transport vector when they reach their appropriate destinations. In some cases, these unloaded macromolecules may subsequently be transferred to still other compartments.

It is known, at least in general terms, that different axonally transported proteins have different sites of deposition within neurons (Goodrum and Morell, 1982; Toews et al., 1982; Stone et al., 1984; Rulli and Wilson, 1987). Possible signals at the molecular level that might account for the selective targeting of transported proteins include "address sequences" analogous to those involved in sorting and intracellular routing in other systems (for reviews, see Blobel, 1983; Wickner and Lodish, 1985) or posttranslational modifications involving sulfation or glycosylation (Hammerschlag, 1983/84; Stone et al., 1984).

Phospholipids and cholesterol are transported as part of the same membranous transport vesicles as proteins (Grafstein et al., 1975; Longo and Hammerschlag, 1980; for review, see Ledeen, 1985). Questions arise as to whether various classes of axonally transported lipids are differentially unloaded along axons, and whether there is selective targeting for axons or nerve endings. We have previously reported differences in the kinetics of anterograde axonal transport for individual lipid classes that are consistent with marked differences in the degree of their unloading during transport (Toews et al., 1979, 1983; Blaker et al., 1980, 1981). We now report results of biochemical and autoradiographic studies characterizing the unloading of axonally transported ethanolamine and choline phosphoglycerides from the transport vector, as well as the subsequent transfer of these lipids to other compartments. A physical model is proposed to account for our results, since lipids do not have the same potential for "target signals" as do proteins.

\section{Materials and Methods}

Injection of radioactive precursors. The procedures for injection of radioactive precursors have been described previously (Armstrong et al., 1985a). Briefly, adult male Long-Evans rats were placed under ketamine/xylazine anesthesia and a dorsal laminectomy was performed to expose the L5 dorsal root ganglion (cell bodies of sciatic nerve sensory neurons) or the spinal cord at the level of L5 dorsal root insertion (cell bodies of sciatic nerve motor neurons in ventral horn region). Radioactive precursor, consisting of $200 \mu \mathrm{Ci}$ of either $2 \cdot{ }^{3} \mathrm{H}$-glycerol $(10 \mathrm{Ci} /$ mmol; ICN, Irvine, CA), $1{ }^{3} \mathrm{H}$-ethanolamine $(30 \mathrm{Ci} / \mathrm{mmol}$; Amersham, Arlington Heights, IL), or methyl- ${ }^{3} \mathrm{H}$-choline $(90 \mathrm{Ci} / \mathrm{mmol}$; Amersham) in $1 \mu \mathrm{l}$ of sterile $0.9 \%$ saline was then injected into either the ganglion or the spinal cord ventral horn, using a pulled capillary micropipette secured in a micromanipulator. Following injection, the surgical field was flushed with sterile saline solution and packed with Gelfoam prior to closure. Rats were kept on $37^{\circ} \mathrm{C}$ isothermal pads (Deltaphase Pads; 

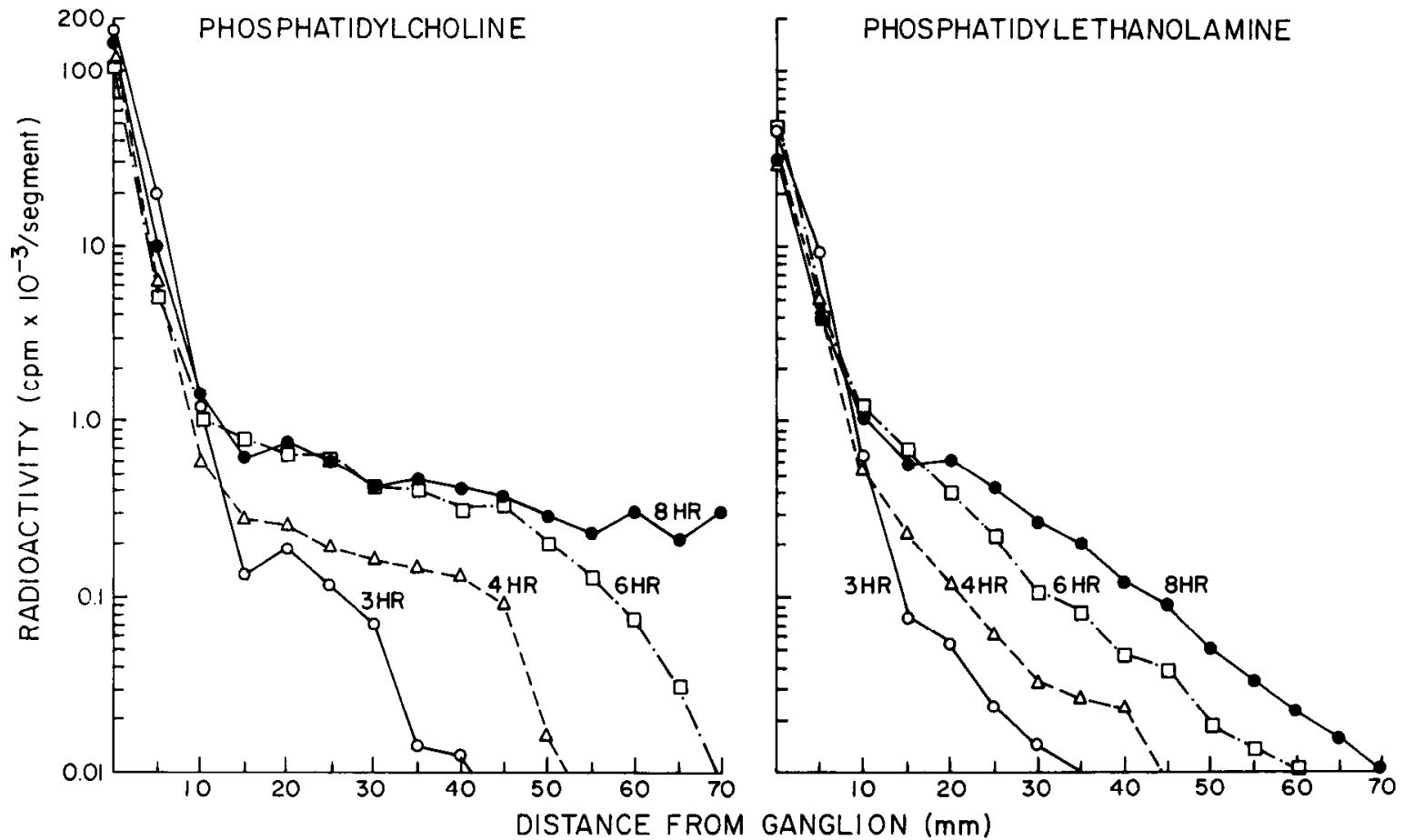

Figure 1. Transport profiles for $2-{ }^{3} \mathrm{H}$-glycerol-labeled phosphatidylcholine and phosphatidylethanolamine in rat sciatic nerve. Nerves were dissected at various times following injection of precursor into the L5 dorsal root ganglion and sectioned into 5 mm segments. Lipids were then extracted, separated by thin-layer chromatography, and the radioactivity present in individual lipid classes determined by liquid-scintillation counting. Lipid radioactivity is plotted on the ordinate in a logarithmic scale.

Braintree Scientific, Braintree, MA) to maintain body temperature during recovery.

Transport profle experiments. Rats were killed at various times following injection of $2-{ }^{3} \mathrm{H}$-glycerol into one $\mathrm{L} 5$ dorsal root ganglion. The injected dorsal root ganglion and corresponding sciatic nerve were dissected, sectioned into $5 \mathrm{~mm}$ segments, freeze-dried, and stored at $-70^{\circ} \mathrm{C}$ until analysis.

Clearance experiments. Six hours after injection of $2-{ }^{3} \mathrm{H}$-glycerol into both L5 dorsal root ganglia, rats were again placed under anesthesia and a 5.0 silk ligature was applied to the right sciatic nerve $5 \mathrm{~mm}$ distal to the injected dorsal rot ganglion. Animals were killed at times ranging from 1 to $4 \mathrm{hr}$ later. Both (ligated and nonligated) sciatic nerves, along with their injected dorsal root ganglia, were dissected and sectioned into $5 \mathrm{~mm}$ segments. All samples were freeze-dried and stored at $-70^{\circ} \mathrm{C}$ until analysis.

Motor neuron experiments. Rats were killed at various times after unilateral injection of $2-{ }^{3} \mathrm{H}$-gylcerol into the ventral horn region of the spinal cord. The injected spinal cord region, the L5 spinal roots, and the corresponding sciatic nerve were dissected and sectioned into $5 \mathrm{~mm}$ segments. All samples were freeze-dried and stored at $-70^{\circ} \mathrm{C}$ until analysis.

Analysis of tissue samples. Lipids were extracted from the freeze-dried tissue samples using a modification (Benjamins et al., 1976) of the method of Folch et al. (1957). Radioactivity in individual phospholipid classes was quantitated (Toews et al., 1983) following separation by thin-layer chromatography using $0.25 \mathrm{~mm}$ silica gel $\mathrm{G}$ plates in a solven system containing chloroform/methanol/ammonium hydroxide $(100$ $40: 6, \mathrm{vol} / \mathrm{vol} / \mathrm{vol}$ ). When ${ }^{3} \mathrm{H}$-ethanolamine was used as precursor, 2-dimensional thin-layer chromatography with an intermediate acidhydrolysis step (Horrocks and Sun, 1972) was used to separate phosphatidylethanolamine (diacyl form) from phosphatidalethanolamine (plasmalogen form).

Autoradiographic experiments. Radioactive precursor $(200 \mu \mathrm{Ci}$ of either ${ }^{3} \mathrm{H}$-choline or ${ }^{3} \mathrm{H}$-ethanolamine) was injected into one $\mathrm{L} 5$ dorsal root ganglion to label sciatic nerve sensory neurons. At vàrious times following precursor injection ( $8 \mathrm{hr}, 1 \mathrm{~d}$, or 1 week), rats were perfused through the aorta with $4 \%$ paraformaldehyde followed by $4 \%$ glutaraldehyde (both in $0.1 \mathrm{~m}$ Sorenson's phosphate buffer). Sciatic nerve segments $15,30,45$, and $60 \mathrm{~mm}$ distal to the injected ganglion were then dissected and processed for lipid autoradiography as described previously (Gould and Dawson, 1976; Gould and Sinatra, 1981).

Light-microscopic autoradiographic analysis was performed on micrographs of sciatic nerve cross sections that were enlarged $1500 \times$. The density of grains in various structural compartments (axon, axon/myelin interface, myelin) was quantitated from the number of grains in a given compartment divided by the relative area of that compartment. The relative area was taken to be the relative frequency at which intersections of a square-lattice grid overlay fell over a given compartment.

\section{Results}

\section{Axonal transport profiles for phosphatidylcholine and phosphatidylethanolamine}

The distribution of labeled phosphatidylcholine and phosphatidylethanolamine along sensory axons of sciatic nerve was examined at various times following injection of $2-{ }^{3} \mathrm{H}$-glycerol into the L5 dorsal root ganglion (Fig. 1). Transport profiles for phosphatidylcholine (one of the 2 major transported phospholipid classes) showed a well-defined initial crest of transported radioactivity moving down the nerve, followed by a relatively flat plateau (Fig. 1), suggesting only a small degree of unloading during transport. In contrast, transport profiles for phosphatidylethanolamine (the other major transported phospholipid class) did not show a distinct crest; rather, the profile was attenuated as it moved down the nerve, suggesting extensive unloading of labeled phosphatidylethanolamine from the moving transport vector. This unloading presumably results from an exchange between transported labeled phosphatidylethanolamine and unlabeled phosphatidylethanolamine in stationary axonal structures, rather than from a net deposition of phosphatidylethanolamine. Diffusion of lipids is not a factor in establishing these transport profiles; colchicine ( $12 \mu \mathrm{g}$ injected intraneurally $4 \mathrm{~mm}$ distal to the L5 dorsal root ganglion) inhibited incorporation of 


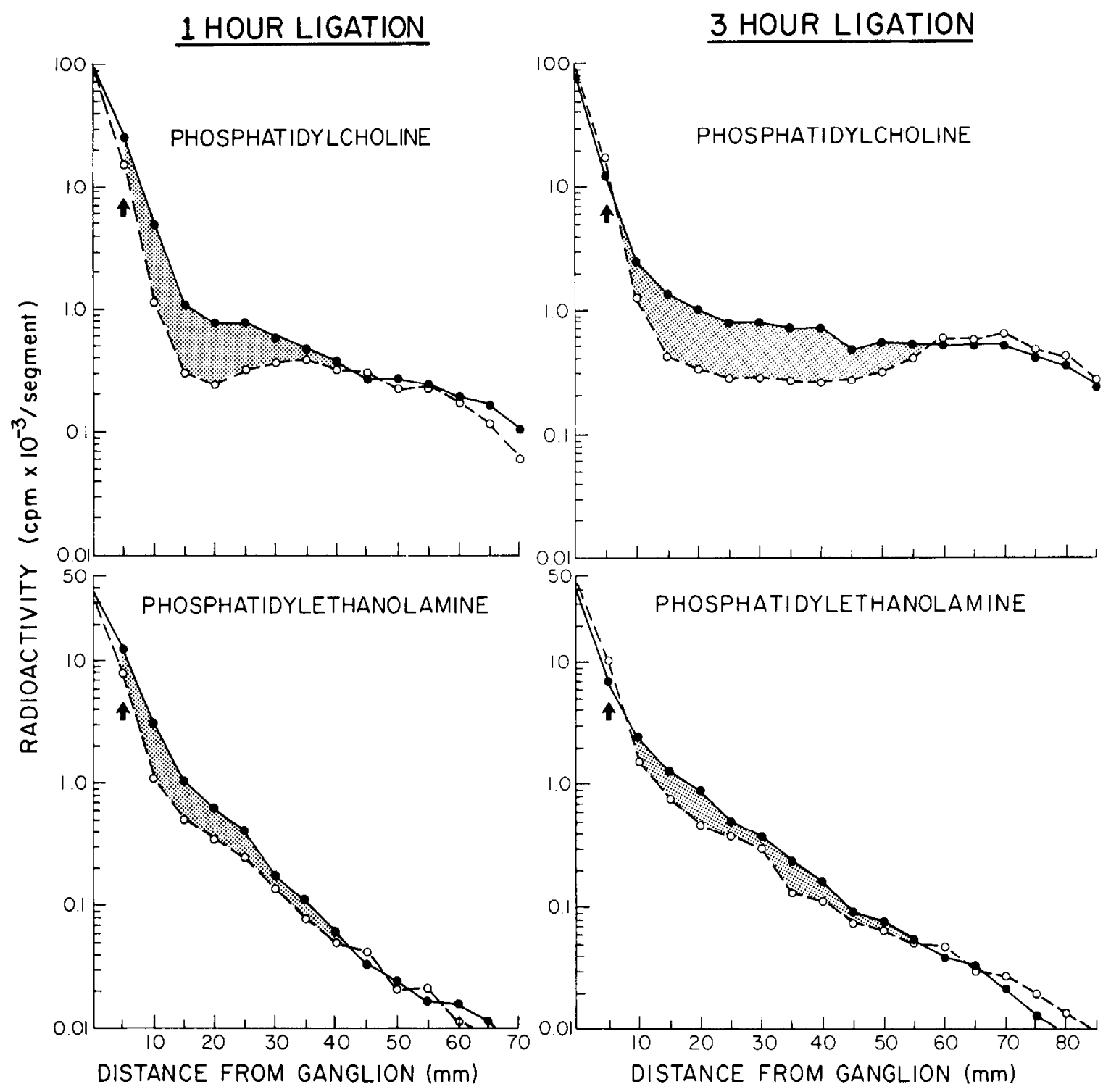

Figure 2. Clearance of axonally transported phosphatidylcholine and phosphatidylethanolamine distal to a ligature on the sciatic nerve. $2-{ }^{3} \mathrm{H}-g l y c e r o l$ was injected into both L5 dorsal root ganglia and axonal transport allowed to proceed for $6 \mathrm{hr}$. A ligature was then placed on one nerve; after 1 $\mathrm{hr}$ (left panels) or $3 \mathrm{hr}$ (right panels), both nerves were dissected and analyzed as described in Figure l. Nonligated control nerve (- - ); ligated nerve $(0--O)$. The arrow indicates the position of the ligature, and the shaded area represents lipids cleared from the nerve distal to the ligature.

$2-{ }^{3} \mathrm{H}$-glycerol into lipids by only $15 \%$, but depressed axonal transport of both phosphatidylcholine and phosphatidylethanolamine by about $85 \%$.

\section{Clearance experiments}

In order to test whether differences in the transport profiles for phosphatidylcholine and phosphatidylethanolamine are due to differences in the degree to which they are unloaded during transport, we compared their clearance distal to a ligature placed on the sciatic nerve (Fig. 2). Transport profiles for phosphatidylcholine in the ligated nerve showed a depression distal to the ligature, after which a relatively flat plateau resumed. This depression broadened with increasing ligation time in a manner consistent with the rapid rate of anterograde axonal transport. Although the transport profiles for phosphatidylethanolamine in ligated nerve also became displaced with time, the shapes remaincd very similar to those in control (nonligated) nerves.
These data indicate that much of the labeled phosphatidylcholine in the nerve was in transit, while most labeled phosphatidylethanolamine in the nerve had already exchanged with stationary axonal lipids.

\section{Axonal transport in motor neurons}

Differences in the extent of unloading of phosphatidylcholine and phosphatidylethanolamine in sensory neurons of sciatic nerve could be due to differences in the behavior of axonally transported lipids within myelinated versus unmyelinated axons, since injection of precursor into the dorsal root ganglion labels both myelinated and unmyelinated sensory fibers. We therefore examined transport profiles for these 2 lipids in sciatic nerve motor neurons (a relatively homogeneous population of myelinated fibers), using $2{ }^{3} \mathrm{H}$-glycerol as precursor. Transport profiles for phosphatidylcholine and phosphatidylethanolamine in this system ( $8 \mathrm{hr}$ profiles; see Fig. 3 ) were very similar to 


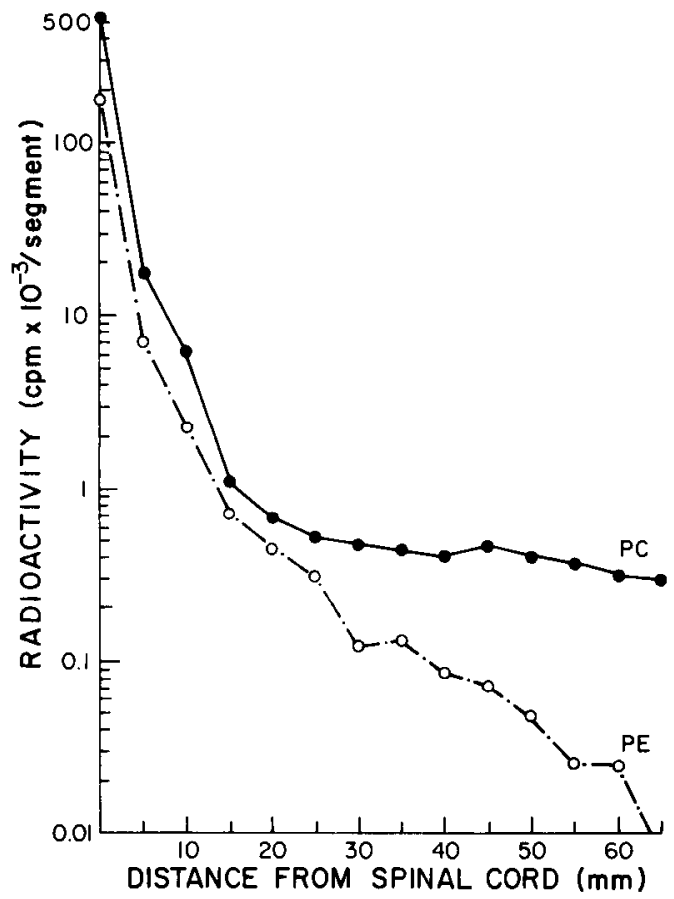

Figure 3. Transport profiles for phosphatidylcholine $(P C)$ and phosphatidylethanolamine $(P E)$ in motor fibers of sciatic nerve at $8 \mathrm{hr}$ following injection of $2-{ }^{3} \mathrm{H}$-glycerol into the ventral horn region of the spinal cord. Data were analyzed and plotted as described in Figure 1.

those obtained when sensory neurons were examined (Fig. 1), indicating no marked differences in the degree of unloading between myelinated and unmyelinated axons.

\section{Transfer of unloaded lipids to other compartments}

We used an autoradiographic approach to determine whether axonally transported choline or ethanolamine phosphoglycerides were differentially transferred to other structural compartments in the nerve subsequent to their unloading from the transport vector. This experimental approach requires that these phospholipid classes be labeled separately (i.e., in their base moieties) and, further, that transport profiles for these baselabeled lipids be similar to those obtained with $2-{ }^{3} \mathrm{H}-$ glycerol. As shown in Figure 4, transport profiles for base-labeled ethanolamine and choline phosphoglycerides in sciatic nerve sensory fibers are similar to those previously obtained for these lipids with $2-{ }^{3} \mathrm{H}$-glycerol as precursor (Fig. 1). Note that there is one complication with this experimental design: ${ }^{3} \mathrm{H}$-ethanolamine labels both phosphatidylethanolamine and ethanolamine plasmalogens (phosphatidalethanolamine). This was not the case with respect to the previous experiments using $2-{ }^{3} \mathrm{H}$-glycerol, since that precursor does not significantly label plasmalogens (Hajra, 1969; Wykle and Snyder, 1969). Transport profiles for phosphatidylethanolamine and ethanolamine plasmalogens were very similar to each other (Fig. 4), with both showing attenuation of the crest of transported radioactivity. This suggests that both of these classes of ethanolamine phosphoglycerides are unloaded to similar degrees during transport. An additional consideration with this experimental design is that the base label should be incorporated and retained in the phospholipid classes being examined. Virtually all ( $>95 \%$ ) of the ${ }^{3} \mathrm{H}$-ethanolamine lipid label remained in ethanolamine phosphoglycerides throughout the 1 week time period examined, while phosphatidylcholine ac-

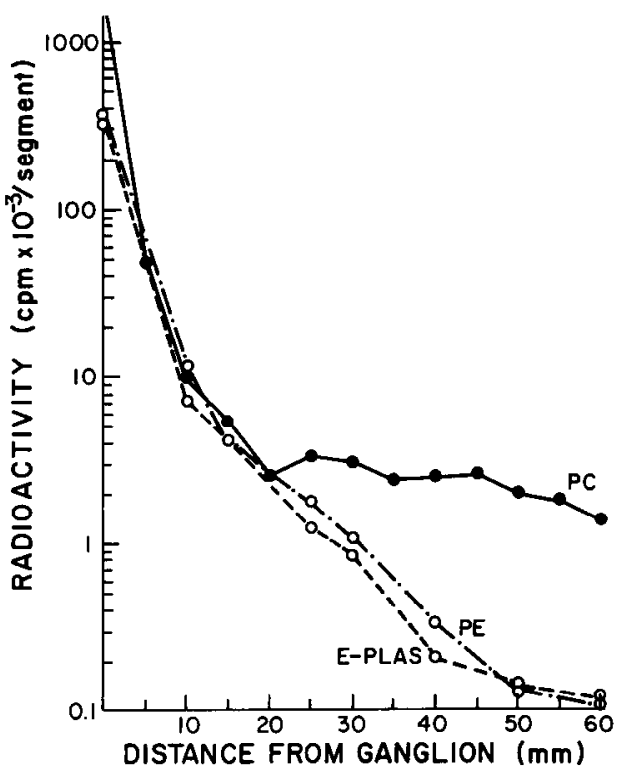

Figure 4. Transport profiles for phosphatidylethanolamine $(P E)$, ethanolamine plasmalogens $(E-P L A S)$, and phosphatidylcholine $(P C)$ at 8 hr following injection of ${ }^{3} \mathrm{H}$-ethanolamine (PE and E-PLAS) or ${ }^{3} \mathrm{H}-$ choline (PC) into the L5 dorsal root ganglion. PE and E-PLAS were separated by 2-dimensional thin-layer chromatography with an intermediate acid-hydrolysis step (Horrocks and Sun, 1972).

counted for $85-95 \%$ of the total ${ }^{3} \mathrm{H}$-choline lipid label.

Quantitation of autoradiographic results (Fig. 5) was restricted to large myelinated fibers, where grains over the axon, axon/ myelin interface, and myelin compartments could be clearly distinguished. For ethanolamine-labeled lipids at $8 \mathrm{hr}$, it was only possible to obtain autoradiographic data for the nerve segment $15 \mathrm{~mm}$ distal to the ganglion, since radioactivity levels in more distal segments were too low to allow accurate quantitation (as expected from data in the transport profiles; see Fig. 1). With both precursors, distinct radial gradients (axon-axon/myelin interface-myelin) were present at an early time point $(8 \mathrm{hr})$, with most of the label present in the axon and axon/myelin interface compartments. These radial gradients were more extreme distally, where labeled lipids were just arriving, than in more proximal segments, where the outward transfer of unloaded lipids was already apparent (for example, compare $8 \mathrm{hr}$ choline distribution at 15 and $45 \mathrm{~mm}$, and $1 \mathrm{~d}$ ethanolamine distribution at 15 and $60 \mathrm{~mm}$ ). These gradients dissipated with increasing time after injection, reflecting a continuous outward equilibration of unloaded labeled lipids.

It is noteworthy that at an early time point $(8 \mathrm{hr})$, most of the ethanolamine-labeled lipids were retained in intra-axonal structures, with very little localized in myelin, while, in comparison, a somewhat larger portion of the choline-labeled lipids rapidly appeared in myelin. By 1 week, a significant amount of label from both precursors was present in myelin, relative to the other 2 compartments examined (Fig. 6). However, the amount of labcl retaincd in myclin at 1 wcck is actually quitc small, compared to the total amount of labeled lipid that has been transported down the axons during this time interval.

\section{Discussion}

In the present study, we provide data that support the hypothesis that various classes of axonally transported lipids are unloaded to differing degrees during their movement down the axons. 

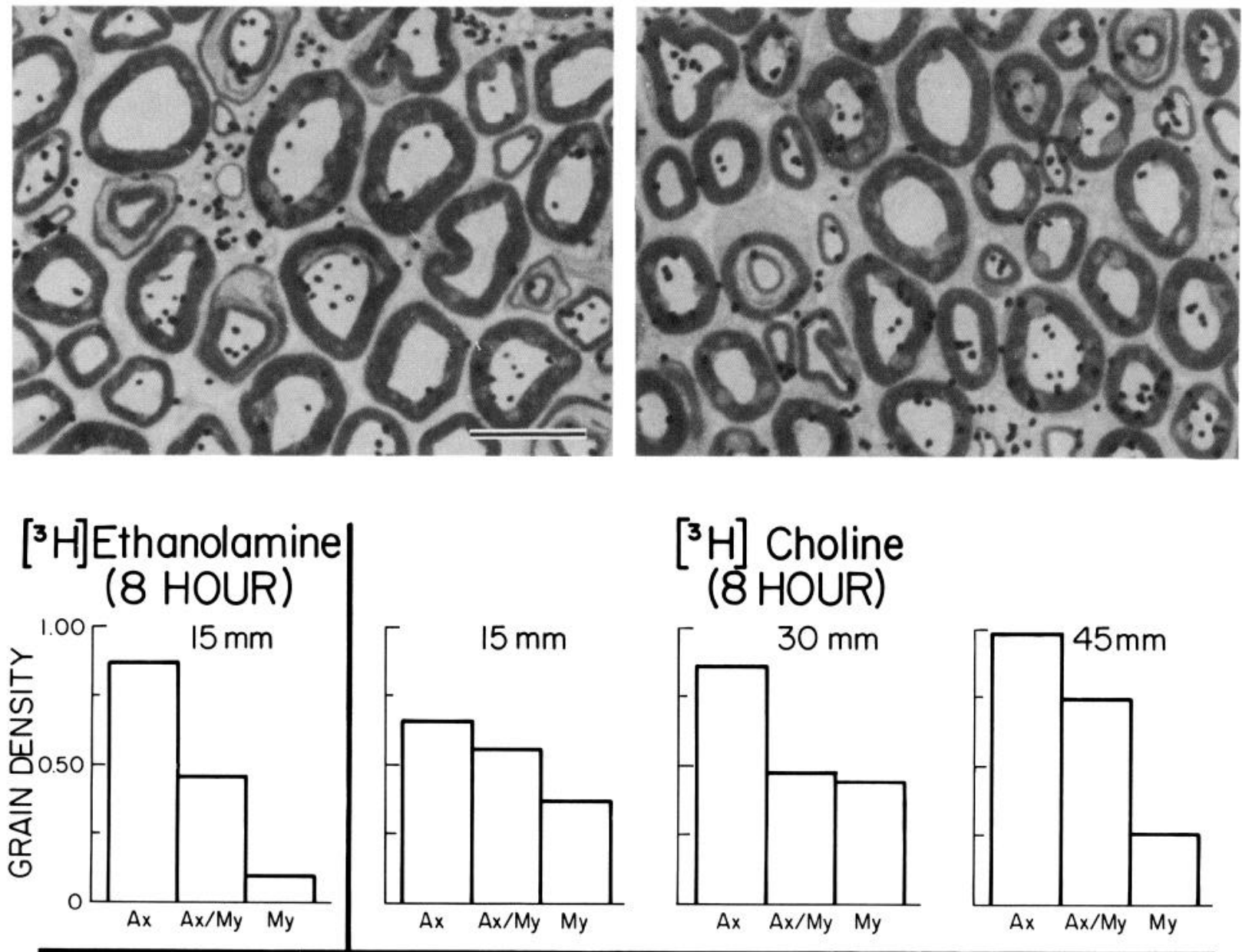

\section{$\left[{ }^{3} \mathrm{H}\right]$ Ethanolamine (I DAY)}
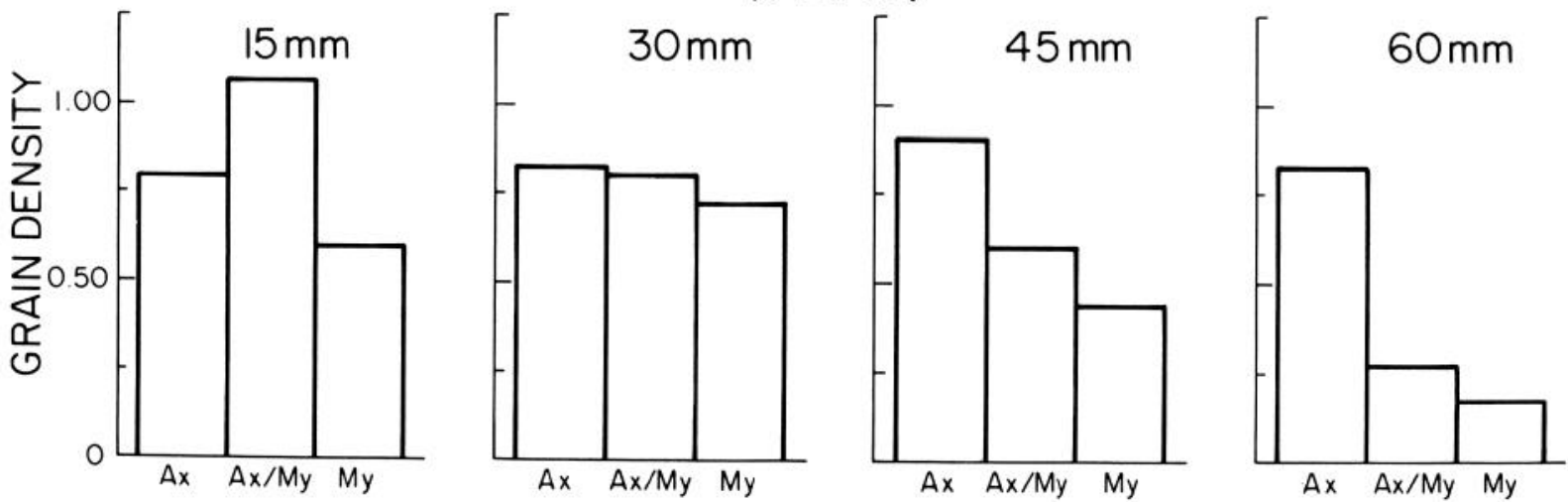

Figure 5. Autoradiographic localization of axonally transported ethanolamine and choline phosphoglycerides. Top, Photomicrographs of sciatic nerve $15 \mathrm{~mm}$ from the injected L5 dorsal root ganglion, illustrating the distribution of silver grains $8 \mathrm{hr}$ following injection of ${ }^{3} \mathrm{H}$-ethanolamine (left) or ${ }^{3} \mathrm{H}$-choline (right) into the L5 dorsal root ganglion. Bar, $10 \mu \mathrm{m}$. Bottom, Quantitative distribution of silver grains over axons, the axon/ myelin interface, and myelin in large myelinated fibers of sciatic nerve. The grain density is the number of grains counted over a structural compartment, divided by the relative area of that compartment. Note that unmyelinated fibers are also labeled.

This unloading probably occurs via exchange of transported labeled lipid for unlabeled lipid in stationary axonal structures. We interpret our data as indicating that transported ethanolamine phosphoglycerides exchange extensively with stationary axonal lipid, while, in contrast, transported phosphatidylcholine exchanges to a much smaller degree, with most continuing to move down the axons. Transport profile differences for these phospholipid classes are similar in sensory fibers (unmyelinated and myelinated axons) and in motor fibers (more heavily myelinated axons). These differences thus cannot be attributed to 

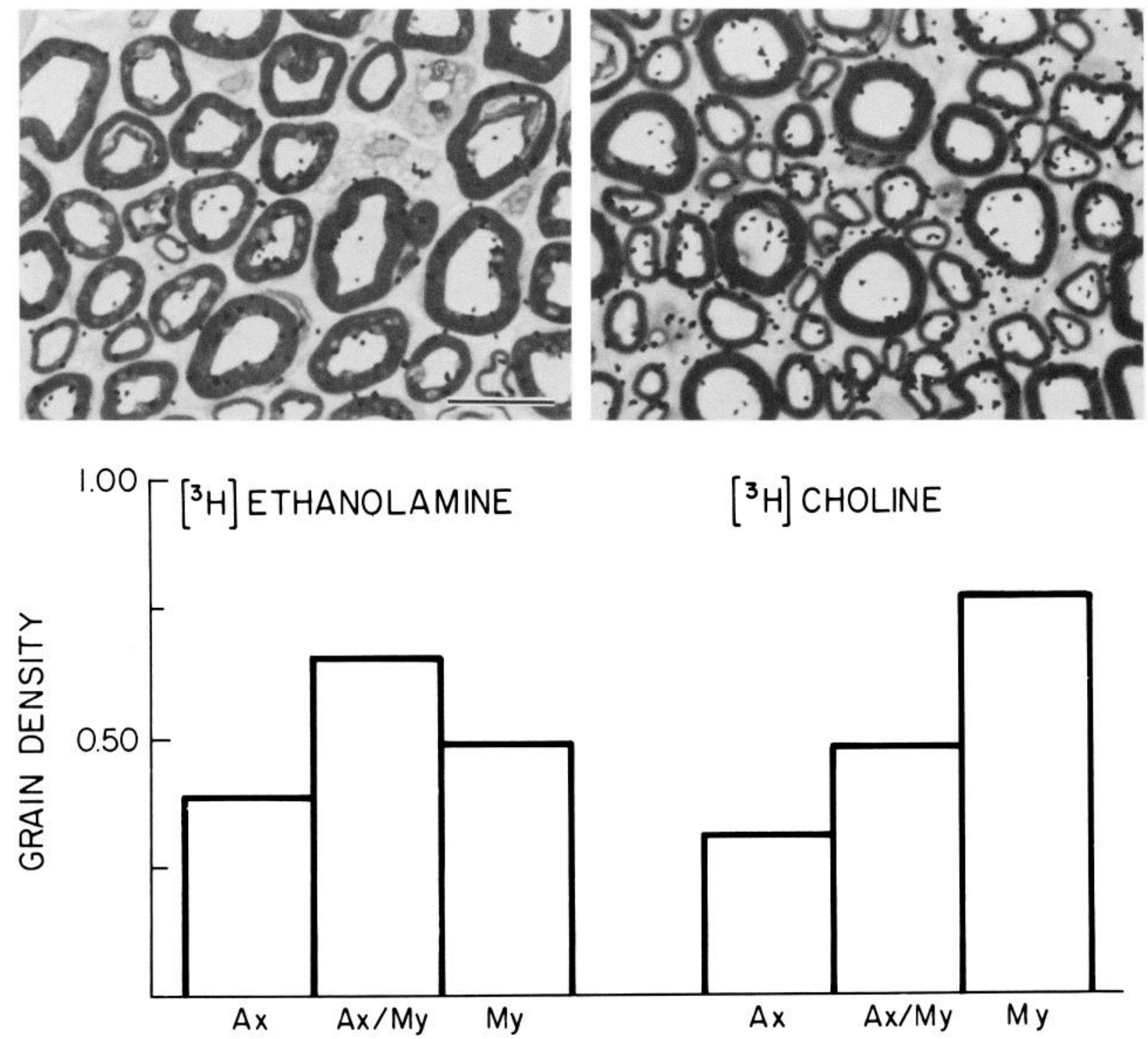

Figure 6. Autoradiographic localization of axonally transported ethanolamine and choline phosphoglycerides 1 week after precursor injection. Top, Photomicrographs of sciatic nerve $15 \mathrm{~mm}$ distal to the injected $\mathrm{L} 5 \mathrm{dorsal}$ root ganglion (left, ${ }^{3} \mathrm{H}$-ethanolamine precursor; right, ${ }^{3} \mathrm{H}$-choline precursor). Bar, $10 \mu \mathrm{m}$. Bottom, Quantitative distribution of silver grains, determined as described in Figure 5, in nerve segments 15 mm distal to the injected ganglion. Note that unmyelinated fibers are also labeled.

a differential behavior of transported lipids in myelinated versus unmyelinated fibers.

The lack of a well-defined crest for transported ethanolamine phosphoglycerides could conceivably result from heterogeneous transport velocities of organelles containing this lipid. However, in order to account for the differences between the shapes of the transport profiles for phosphatidylcholine and ethanolamine phosphoglycerides, such a hypothesis would require that organelles in the initial crest contain labeled phosphatidylcholine but little or no labeled ethanolamine phosphoglycerides, and conversely, that other vesicles moving at slower and varied rates contain labeled ethanolamine phosphoglycerides but little or no labeled phosphatidylcholine. These 2 phospholipid classes are both present in significant quantities in all nervous system membranes and subcellular fractions for which the composition has been determined (for reviews, see Rouser et al., 1972; Ansell, 1973; Porcellati and Arienti, 1983; DeVries, 1984; Whittaker,
1984), and this is true of biological membranes in general as well (McMurray, 1973; White, 1973; Ansell and Spanner, 1982). Thus, we do not consider the segregation of these 2 major membrane lipids into discrete populations of transport vesicles to be a likely explanation for differences in transport profiles for phosphatidylcholine and ethanolamine phosphoglycerides.

Our autoradiographic results showing the location of axonally transported lipids are in general agreement with those reported for the chick oculomotor system by Droz and co-workers (for review, see Droz et al., 1985). In the present study, labeled ethanolamine phosphoglycerides that were unloaded from the transport vector were initially retained in axonal structures; some of this unloaded lipid was subsequently transferred to the axon/ myelin interface (axolemma?), and some eventually further equilibrated with bulk myelin. Although transported phosphatidylcholine was not extensively unloaded, a small subset of transported phosphatidylcholine, perhaps consisting of a spe- 


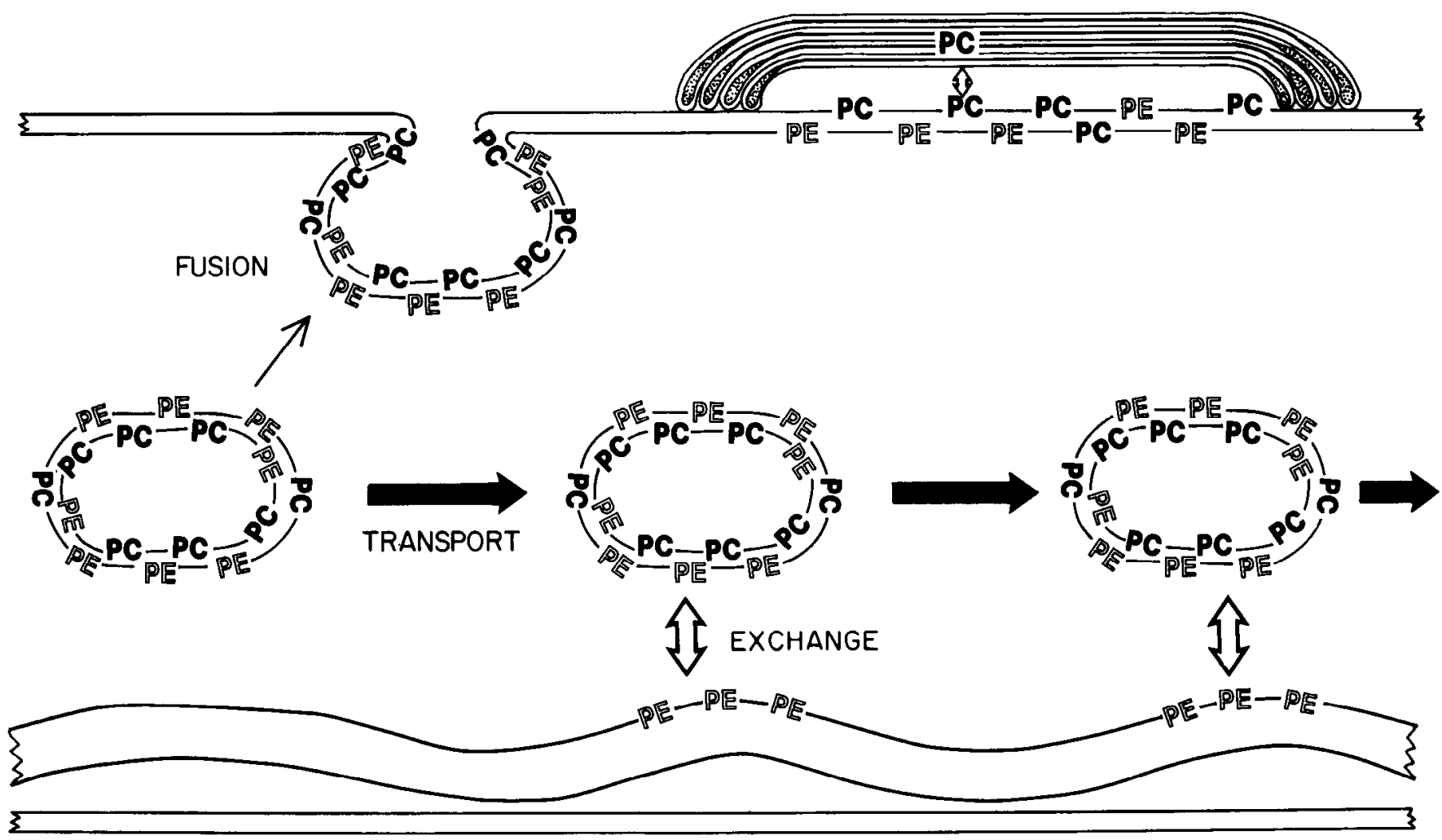

Figure 7. Schematic diagram of a proposed physical model to explain differential unloading of axonally transported phospholipids. According to this model, axonally transported phospholipids are asymmetrically distributed across the membrane bilayer of the transport vesicles, with phosphatidylcholine $(P C)$ enriched in the inner leaflet, and ethanolamine phosphoglyccridcs (phosphatidyl- and phosphatidalethanolamine; $P E$ ) preferentially localized in the outer leaflet. During transport, $P E$ is continually available for extensive exchange with lipids in stationary axonal structures, while, in contrast, less PC is available for exchange. In addition to the bulk movement of transport vesicles down the axons and the accompanying extensive exchange of $\mathrm{PE}$, a small portion of transport vesicles is destined for fusion with the axolemma. This fusion process, with accompanying inversion of original transport vesicle topography, thus delivers a small pool of PC directly to the outer leaflet of the axolemmal membrane, where it is preferentially (relative to PE) available for subsequent transfer to the surrounding myelin.

cialized pool (see below), did appear to be rapidly transferred to myelin. An additional fraction of the transported phosphatidylcholine was also unloaded and sequentially transferred radially with a time course similar to that for labeled ethanolamine phosphoglycerides. However, the transfer of all of these axonally transported lipid classes from axons to myelin is a quantitatively minor event compared to the synthesis of large amounts of myelin lipids by the myelin-maintaining glial cells (Droz et al., 1981; Alberghina et al., 1982).

The markedly different behavior of transported choline and ethanolamine phosphoglycerides suggests that the specificity for phospholipid unloading is defined by the base moiety. This interpretation is consistent with our observation that both the diacyl and plasmalogen forms of the ethanolamine phosphoglycerides are unloaded to similar degrees along axons. Furthermore, subcellular fractionation studies in the optic pathway (Alberghina et al., 1985) and sciatic nerve (Toews et al., 1984; Armstrong et al., 1985b) indicate that neither form of ethanolaminc phosphoglyceride is selcctively transferred to myelin, as was suggested previously by Brunetti et al. (1983).

The actual mechanisms involved in unloading axonally transported phospholipids (transfer from the transport vector to some stationary axonal structure) are as yet unknown. The involvement of soluble lipid transfer or exchange proteins in this process is one possibility (for discussion, see Ledeen, 1985), and the preferential involvement of specific transfer proteins might par- tially explain differences in the degree of unloading of individual lipid classes. Some lipids may also be unloaded because of their close association with specifically targeted proteins, and a preferential association of lipid classes with such proteins might also account for minor differences in lipid unloading. Another possibility is that there may be actual contact between the membranous transport vector and stationary membranes in the axons, with subsequent differential degrees of unloading (exchange) for various lipids.

Figure 7 is a schematic diagram of a proposed physical model, consistent with our experimental results, wherein an asymmetric distribution of phospholipid classes across the membrane bilayer of the transport vector plays a role in this differential exchange process. According to this model, phosphatidylcholine is enriched in the inner leaflet of the membrane bilayer of the transport vector, and undergoes minimal trans-bilayer movement, as suggested by evidence in other systems (Sleight and Pagano, 1984, 1985). If this were the case, transported phosphatidylcholine would be unlikely to exchange extensively with lipid in other intra-axonal structures during transport. However, that subset of the transport vesicles destined for insertion into axolemma (presumably by vesicle fusion, with subsequent inversion of the original topography; see upper portion of Fig. 7) might deliver a small pool of phosphatidylcholine directly to the outer leaflet of the axolemmal membrane. Fusion would thus render this small pool readily available for subsequent rapid 
exchange with the inner myelin layers, and might explain the early appearance of some labeled phosphatidylcholine in myelin. The preferential localization of phosphatidylcholine on the outer leaflet of axolemmal membrane has been previously suggested by Brunetti et al. (1981) as a possible explanation for the apparent ready movement of this lipid into the inner myelin layers, which was noted in their study as well. In contrast to phosphatidylcholine, ethanolamine phosphoglycerides may be enriched in the outer leaflet of the membrane bilayer of the transport vector; in addition, these lipids have been shown to readily undergo rather extensive trans-bilayer movement ( $\mathrm{Pa}$ gano and Sleight, 1985; Sleight and Pagano, 1985). Either or both of these factors might thus render transported ethanolamine phosphoglycerides readily available for more extensive exchange with the large pool of lipids comprising various intraaxonal structures, as well as with the axolemma. Thus, we suggest that differences in the topographical orientation of various lipid classes in the lipid bilayer of the transport vector may play a role in modulating the degree of unloading during transport.

The mechanisms responsible for transfer of axonally transported lipids to other compartments subsequent to their unloading also remain largely unknown. Equilibration of intact axolemmal lipids with those in inner myelin layers by simple diffusion and exchange is one possibility. However, Alberghina et al. (1985) noted that the acyl-chain-length pattern of axonally transported phospholipids transferred to myelin differs from that of phospholipids remaining in axons, and suggested that some "remodeling" process was involved in controlling the transfer of lipids from axons to myelin. In addition, some redistribution of label may occur owing to degradation of transported lipid, with transfer of free labeled precursor to myelin and subsequent reincorporation of label (for further discussion, see Ledeen, 1985).

Studies of the processes involved in the unloading and subsequent transfer of axonally transported lipids, and of how these processes are controlled, provide insights into the mechanisms involved in maintaining the functional integrity of axons and nerve endings; such studies may also be of relevance to a better understanding of mechanisms involved in membrane maintenance in other cell types as well.

\section{References}

Alberghina, M., M. Viola, and A. M. Giuffrida (1982) Transfer of axonally transported phospholipids into myelin isolated from rabbit optic pathway. Neurochem. Res. 7: 139-149.

Alberghina, M., M. Viola, F. Moro, and A. M. Giuffrida (1985) Remodeling and sorting process of ethanolamine and choline glycerophospholipids during their axonal transport in the rabbit optic pathway. J. Neurochem. 45: 1333-1340.

Ansell, G. B. (1973) Phospholipids and the nervous system. In Form and Function of Phospholipids, 2nd Ed., G. B. Ansell, J. N. Hawthorne, and R. M. C. Dawson, eds., pp. 377-422, Elsevier, Amsterdam.

Ansell, G. B., and S. Spanner (1982) Phosphatidylserine, phosphatidylethanolamine and phosphatidylcholine. In Phospholipids, J. N. Hawthorne and G. B. Ansell, eds., pp. 1-49, Elsevier, Amsterdam.

Armstrong, R., A. D. Toews, R. B. Ray, and P. Morell (1985a) Retrograde axonal transport of endogenous phospholipids in rat sciatic nerve. J. Neurosci 5: 965-969.

Armstrong, R., A. D. Toews, R. B. Ray, P. Morell, and R. M. Gould (1985b) The transfer of ethanolamine phosphatides from axon to myelin (Abstr.). Trans. Am. Soc. Neurochem. 16: 134.

Benjamins, J. A., S. Miller, and P. Morell (1976) Metabolic relationships between myelin subfractions: Entry of galactolipids and phospholipids. J. Neurochem. 27: 565-570.

Blaker, W. D., A. D. Toews, and P. Morell (1980) Cholesterol is a component of the rapid phase of axonal transport. J. Neurobiol. 11: 243-250.

Blaker, W. D., J. F. Goodrum, and P. Morell (1981) Axonal transport of the mitochondria-specific lipid, diphosphatidylglycerol, in the rat visual system. J. Cell Biol. 89: 579-584.

Blobel, G. (1983) Regulation of intracellular protein traffic. In Molecular and Cellular Interactions (Progress in Brain Research, vol. 58), J.-P. Changeux, J. Glowinski, M. Imbert, and F. E. Bloom, eds., pp. 77-93, Elsevier, Amsterdam.

Brunetti, M., L. DiGiamberardino, G. Porcellati, and B. Droz (1981) Contribution of axonal transport to the renewal of myelin phospholipids in peripheral nerves. II. Biochemical study. Brain Res. 219: 73-84.

Brunetti, M., B. Droz, L. DiGiamberardino, H. L. Koenig, F. Carretero, and G. Porcellati (1983) Axonal transport of ethanolamine glycerophospholipids: Preferential accumulation of transported ethanolamine plasmalogen in myelin. Neurochem. Pathol. 1: 59-80.

DeVries, G. H. (1984) The axonal plasma membrane. In Structural Elements of the Nervous System (Handhook of Neurochemistry, vol. 7, 2nd Ed.), A. Lajtha, ed., pp. 341-360, Plenum, New York.

Droz, B., L. DiGiamberardino, and H. L. Koenig (1981) Contribution of axonal transport to the renewal of myelin phospholipids in peripheral nerves. I. Quantitative radioautographic study. Brain Res. 219: 57-71.

Droz, B., M. Brunetti, L. DiGiamberardino, H. L. Koenig, and G. Porcellati (1985) Selective distribution of axonally transported phospholipids to nerve endings and/or myelin: The case of ethanolamine glycerophospholipids. In Phospholipids in the Nervous System, vol. 2: Physiological Roles, L. A. Horrocks, J. N. Kanfer, and G. Porcellati, eds., pp. 315-327, Raven, New York.

Folch, J., M. Lees, and G. H. Sloane Stanley (1957) A simple method for the isolation and purification of total lipids from animal tissues. J. Biol. Chem. 226: 497-509.

Goodrum, J. F., and P. Morell (1982) Axonal transport, deposition and metabolic turnover of glycoproteins in the rat optic pathway. $J$. Neurochem. 38: 696-704.

Gould, R. M., and R. M. C. Dawson (1976) Incorporation of newly formed lecithin into peripheral nerve myelin. J. Cell Biol. 68: 480496.

Gould, R. M., and R. S. Sinatra (1981) Internodal distribution of phosphatidylcholine biosynthetic activity in teased peripheral nerve fibers: An autoradiographic study. J. Neurocytol. 10: 161-167.

Grafstein, B., and D. S. Forman (1980) Intracellular transport in neurons. Physiol. Rev. 60: 1167-1283.

Grafstein, B., J. A. Miller, R. W. Ledeen, J. Haley, and S. C. Specht (1975) Axonal transport of phospholipid in goldfish optic system. Exp. Neurol. 46: 261-281.

Hajra, A. (1969) Biosynthesis of alkyl-ether containing lipid from dihydroxyacetone phosphate. Biochem. Biophys. Res. Commun. 37: 486-492.

Hammerschlag, R. (1983/84) How do neuronal proteins know where they are going? Speculations on the role of molecular address markers. Dev. Neurosci. 6: 2-17.

Horrocks, L. A., and G. Y. Sun (1972) Ethanolamine plasmalogens In Research Methods in Neurochemistry, vol. 1, N. Marks and R. Rodnight, eds., pp. 223-231, Plenum, New York.

Ledeen, R. W. (1985) Transport, exchange, and transfer of phospholipids in the nervous system. In Phospholipids in Nervous Tissues, J. Eichberg, ed., pp. 135-172, Wiley, New York.

Longo, F. M., and R. Hammerschlag (1980) Relation of somal lipid synthesis to the fast axonal transport of protein and lipid. Brain Res. 193: 471-485.

McMurray, W. C. (1973) Phospholipids in subcellular organelles and membranes. In Form and Function of Phospholipids, 2nd Ed., G. B. Ansell, J. N. Hawthorne, and R. M. C. Dawson, eds., pp. 205-252, Elsevier, Amsterdam.

Ochs, S. (1982) Axoplasmic Transport and its Relation to Other Nerve Functions, Wiley, New York.

Pagano, R. E., and R. G. Sleight (1985) Defining lipid transport pathways in animal cells. Science 229: 1051-1057.

Porcellati, G., and G. Arienti (1983) Metabolism of phosphoglycerides. In Metabolism in the Nervous System (Handbook of Neurochemistry, vol. 3, 2nd Ed.), A. Lajtha, ed., pp. 133-161, Plenum, New York.

Rouser, G., G. Kritchevsky, A. Yamamoto, and C. F. Baxter (1972) Lipids in the nervous system of different species as a function of age: 
Brain, spinal cord, peripheral nerve, purified whole cell preparations, and subcellular particulates: Regulatory mechanisms and membrane structure. In Advances in Lipid Research, R. Paoletti and D. Kritchevsky, eds., pp 261-360, Academic, New York.

Rulli, R. D., and D. L. Wilson (1987) Destinations of some fasttransported proteins in sensory neurons of bullfrog sciatic nerve. $J$. Neurochem. 48: 134-140.

Sleight, R. G., and R. E. Pagano (1984) Transport of a fluorescent phosphatidylcholine analog from the plasma membrane to the Golgi apparatus. J. Cell Biol. 99: 742-751.

Sleight, R. G., and R. E. Pagano (1985) Transbilayer movement of a fluorescent phosphatidylethanolamine analogue across the plasma membranes of cultured mammalian cells. J. Biol. Chem. 260: 11461154.

Stone, G. C., R. Hammerschlag, and J. A. Bobinski (1984) Fast axonal transport of tyrosine sulfate-containing proteins: Preferential routing of sulfoproteins toward nerve terminals. Cell. Mol. Neurobiol. 4: 249262.

Toews, A. D., J. F. Goodrum, and P. Morell (1979) Axonal transport of phospholipids in rat visual system. J. Neurochem. 32: 1165-1173.

Toews, A. D., B. F. Saunders, and P. Morell (1982) Axonal transport and metabolism of glycoproteins in rat sciatic nerve. J. Neurochem. 39: 1348-1355.

Toews, A. D., B. F. Saunders, W. D. Blaker, and P. Morell (1983) Differences in the kinetics of axonal transport for individual lipid classes in rat sciatic nerve. J. Neurochem. 40: 555-568.

Toews, A. D., R. Ray, R. Armstrong, and P. Morell (1984) Axonal transport kinetics for ethanolamine phosphoglycerides. Trans. Am. Soc. Neurochem. (Abstr.) 15: 246.

Weiss, D. G. (1982) Axoplasmic Transport, Springer-Verlag, New York.

White, D. A. (1973) The phospholipid composition of mammalian tissucs. In Form and Function of Phospholipids, 2nd Ed., G. B. Ansell, J. N. Hawthorne, and R. M. C. Dawson, eds., pp. 441-483, Elsevier, Amsterdam.

Whittaker, V. P. (1984) The synaptic vesicle. In Structural Elements of the Nervous System (Handbook of Neurochemistry, vol. 7, 2nd Ed.), A. Lajtha, ed., pp. 41-69, Plenum, New York.

Wickner, W. T., and H. F. Lodish (1985) Multiple mechanisms of protein insertion into and across membranes. Science 230:400-407.

Wykle, R. L., and F. Snyder (1969) The gylcerol source for biosynthesis of alkyl glycerol ethers. Biochem. Biophys. Res. Commun. 37: 658662. 